\title{
Nanotechnology as a Tool to help Macromolecules Overcoming Biological Barriers
}

\author{
María José Alonso \\ Dept. Pharmacy and Pharmaceutical Technology, \\ University of Santiago de Compostela (USC), Spain
}

\section{Extended Abstract}

The continuously improved understanding of the biological barriers and the molecular biology associated to pathological conditions is paving the way for a more comprehensive and rational design of protein formulations based on the use of nanotechnology. Our laboratory, with a long-track experience in the formulation of macromolecules using polymer nanoparticles, has significantly contributed to this field. As an example, in the 90's we were the first to report that nanoparticles made of either PLA-PEG or chitosan were efficient vehicles for the transmucosal delivery of proteins and antigens. The result of our subsequent efforts is an array of nanotechnologies that can be used to deliver proteins across mucosal surfaces, and, also, to facilitate their intracellular delivery following parenteral administration.

In my presentation, I will focus on the design of protein carriers that could be used in different therapeutic areas: (i) oral delivery of peptides intended to treat either local or systemic diseases, (ii) delivery of mAb targeted to intracellular onco-proteins, as new oncological treatments, (iii) nanovaccines designed to prevent diseases, i.e. HIV.

Overall, our experience in this field has benefited from integrative approaches adopted by specifically designed consortia. Hopefully, the results of these cooperative efforts will help to accelerate the progress of a rational design of protein-based nanomedicines.

More information about these projects can be found at:

http://www.usc.es/grupos/mjalonsolab/

\section{Acknowledgements:}

The following researchers have contributed to the work in different fields:

Oral protein delivery: Matilde Duran, Eleni Samaridou, Carlos Dieguez, Sulay Tovar, Niu Zhigao and Manuel Santander from the USC, Aloise Mabonzo, from the CEA, France and Patrik Lundquist and Per Artursson from UU, Sweden.

Vaccine delivery: Jose Crecente, Tamara Gómez, Ana Olivera and Dolores Torres from the USC, Africa González from the University of Vigo and Ma Luo and Frank Plummer from University of Manitoba, Canada.

Nose-to-brain delivery: Eleni Samaridou and Vanessa Castro from USC, and Hannah Walgrave and Evgenia Salta from VIBLeuven, Leuven, Belgium.

Delivery of anti-cancer monoclonal antibodies: Ana Cadete, Ana Olivera, Desirée Teijeiro and Dolores Torres from the USC, Spain and Gema Moreno and Angela Molina from the UAM, Spain.

The research activity has been founded the the European Comision FP7 (grant agreement $n^{\circ} 281035$-TRANS-INT), the Horizon 2020 Program (grant agreement \# 646142 - NANOPILOT and grant agreement No. 721058- B-SMART), The National Institutes of Health (NIH) (Grant Number: R01AI111805) and the NanoFar European Doctorate, EMJD NanoFar (grant agreement \# 2012-0028) and the World Cancer Research Organization. 